\title{
Population pharmacokinetics of vancomycin in patients receiving extracorporeal membrane oxygenation
}

\author{
Hussain Mulla' \& Suneel Pooboni² \\ ${ }^{1}$ Centre for Pharmacy Practice Research, and ${ }^{2}$ Department of ECMO, Glenfield Hospital, University Hospitals of Leicester NHS Trust, \\ England
}

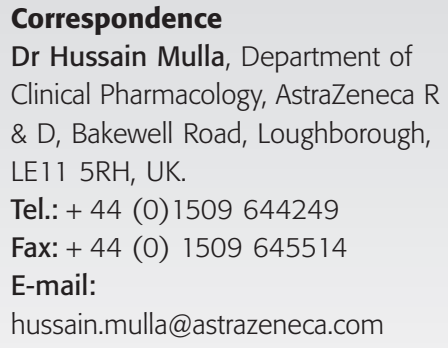

\section{Received}

13 September 2004

Accepted

4 January 2005

\begin{abstract}
Aims
Extracorporeal membrane oxygenation (ECMO) is a life support system used during severe respiratory or cardiorespiratory failure. The objective of this study was to characterize the population pharmacokinetics of vancomycin during ECMO.

Methods

A population model was developed using WinNonMix (Version 2.0.1) from a total of 366 plasma observations in 45 patients, including term neonates, older children and adults. The study utilized both rich prospective and sparse retrospective data. Prospective samples were drawn at baseline and then 30,60,90, 120, 180, 240, 300, 360 and 420 min postinfusion. Steady state concentrations were obtained retrospectively from an assay database, cross-referencing with the patients' medical records.
\end{abstract}

\section{Results}

Data were examined using a two-compartment model with an additive and proportional residual error. Model fit improved substantially when clearance, $\mathrm{CL}\left(\mathrm{kg}^{-1} \mathrm{~h}^{-1}\right)$

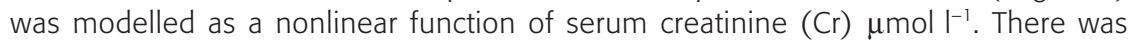
a linear relationship between $\mathrm{CL}$ and age up to 1000 days: $\mathrm{CL}$ (Age < 1000 days) = $[2.4+0.0018 \times$ Age (days) $] / C r ; C L$ (Age $>1000$ days) $=4.3 / \mathrm{Cr}$. Age also influenced central volume $(V 1)$ when included in the model as a dichotomous variable: $V 1$ $($ Age $<4000$ days $)=0.45 \mathrm{I} \mathrm{kg}^{-1} ; V_{1}$ (Age $>4000$ days $)=0.36 \mathrm{I} \mathrm{kg}^{-1}$. Intercompartmental clearance and tissue volume were estimated to be $0.09 \mathrm{l} \mathrm{kg}^{-1} \mathrm{~h}^{-1}$ and $0.25 \mathrm{I} \mathrm{kg}^{-1}$, respectively. Model validation in a separate group of 20 patients revealed a bias of $-7.7 \%$ and a precision of $26.7 \%$.

\section{Conclusions}

The clearance of vancomycin was decreased and its volume of distribution increased in patients receiving $\mathrm{ECMO}$, suggesting altered drug disposition during this treatment.

\section{Introduction}

Extracorporeal membrane oxygenation (ECMO) is a life support system used in the treatment of patients of all ages with severe respiratory or cardiorespiratory failure $[1,2]$. As with other critically ill patients, those with ECMO are at an increased risk of serious infections because of invasive investigations and monitoring, central catheter related bacteraemias, multi-organ failure and immunosuppression. In addition, ECMO patients may have increased risks as a result of prolonged exposure of blood to the large polymeric surface areas of the circulation system and multiple ports of entry for organisms. Vancomycin is administered prophylactically as a single dose immediately postcannulation for ECMO, and also to treat serious infections caused by staphylococci, particularly those that are coagulase-negative. 
Given that maintaining vancomycin concentrations above a minimum threshold is vitally important and that its pharmacokinetics exhibit wide interpatient variability, plasma concentration monitoring is deemed necessary for optimum therapy with vancomycin.

Drug disposition is shown to be significantly altered during ECMO [3]. The expanded circulating volume and sequestration by plastic components of the circuit have been shown to influence the pharmacokinetics of theophylline and midazolam $[4,5]$. In addition, there is a suggestion that recirculation of blood during venovenous cannulation may also contribute to altered drug disposition [4]. The results of previous studies of vancomycin pharmacokinetics during ECMO are conflicting [6-8]. Although an expanded volume of distribution with consequent decreased elimination rate would be anticipated, many of the studies do not corroborate this. However these studies involved neonates or infants and were limited by small numbers. Furthermore, pharmacokinetic parameters were determined using non-population approaches and thus interindividual variability and the influence of significant covariates were not identified satisfactorily. Finally, little or no emphasis was placed on dosage regimen design.

The purpose of this study was to determine the population pharmacokinetics of vancomycin in neonates, older children and adults treated by ECMO. A second aim was, by identifying significant covariates, to establish new dosing guidelines.

\section{Methods}

\section{Patients}

This study was approved by the Leicestershire Research Ethics Committee and utilized both prospective rich and retrospective sparse data. For those patients enrolled in the prospective study, consent was obtained from parents or relatives. All patients in whom vancomycin was administered during ECMO support were eligible for study. Vancomcyin was administered as a $1 \mathrm{~h}$ infusion and initial doses were determined according to age and weight. In the prospective study, plasma samples were taken from those patients administered vancomycin prophylactically as a single dose postcannulation, or after the first and fourth doses when the drug was used to treat suspected or confirmed infection. Samples for analysis were drawn from indwelling arterial lines at baseline prior to initiation of the infusion and then $30,60,90$, $120,180,240,300,360$ and $420 \mathrm{~min}$ postinfusion. Sparse and routine plasma concentration data collected during vancomycin treatment were obtained retrospectively from an assay database held in the Department of Microbiology, cross-referencing with the patients' med- ical records. Only those records where there was a complete set of data were included in the population pharmacokinetic analysis. All concentrations were assumed to be at steady state, usually obtained as peak $(1 \mathrm{~h}$ postinfusion) and trough values (end of dose interval).

The following continuous and categorical clinical data were documented for all patients where possible: age (postnatal, postconceptional and gestational for neonates), gender, weight, diagnosis, duration of ECMO, cannulation mode, outcome, urea, serum creatinine, urine output and requirements for continuous venovenous haemofiltration $(\mathrm{CVVH})$.

\section{Vancomycin analysis}

Vancomycin assays were performed by the Department of Microbiology using the Innofluor $^{\circledR}$ fluorescence polarization immunoassay (Opus Diagnostics Inc, Fort Lee, NJ 07024). The limit of determination was $1 \mathrm{mg}$ $1^{-1}$ and the intra-assay coefficient of variation was less than $6.0 \%$ [9].

\section{Pharmacokinetic analysis}

Rich prospective and sparse retrospective data were collated, evaluated and entered into an Excel spreadsheet, and then analyzed using the mixed effects nonlinear regression modelling programme, WinNonMix Professional (Version 2.0.1) bundled with Compaq Visual Fortran compiler (Professional Edition, Version 6.5) [10, 11]. All modelling was carried out using the First Order Conditional Estimation method.

In the preliminary analysis a one, two or three compartment model with first order elimination was fitted to all data from all subjects simultaneously. Interpatient variability in model parameters was assumed to be log linear, whereas additive, proportional and combined error structures were compared for the residual error on observed concentrations. A diagonal covariance matrix was applied because scatterplots of the Bayesian posterior estimates did not suggest any correlation between the parameters. Scatterplots of covariates against initial parameter estimates were examined to identify those factors that may have a potential influence in the model. Any covariate present in less than $20 \%$ of the population was not tested. Owing to the multicollinearity of several clinical characteristics, body weight was included in the model before evaluation of all other covariates. Allometric scaling transformation of weight $\left(\right.$ Weight $^{0.75}$ ) was also assessed at this point. Following the inclusion of weight, each covariate was then added sequentially to the initial regression model. A multivariate analysis was then performed in a forward stepwise fashion. Covari- 
ates significantly influencing the model were added cumulatively until the best description of the data was obtained. A backward elimination step was then performed by fixing the coefficient of each covariate in turn, to zero. Comparisons of hierarchical models were based on examinations of the graphical diagnostic plots, the precision of parameter estimates and the changes in the interpatient variability as well as the differences in the objective function value (OFV), i.e. two times the negative log likelihood value. A change in the OFV $\geq 7.88$ ( 2 degrees of freedom) was accepted as statistically significant $(P \leq 0.005)$. Covariates were omitted from the model where there was a lack of effect on goodness-of-fit plots or the magnitude of interpatient variability, despite a statistically significant change in OFV. In addition only those covariate relationships that had a plausible physiological basis were included in the final model.

\section{Model validation}

To validate the model and the population pharmacokinetic parameter estimates, plasma concentrations of vancomycin observed during routine monitoring in a separate group of 20 patients were compared with the concentrations predicted by the model. A measure of the predictive performance of models can be determined by calculating the median prediction error percent, i.e. observed minus predicted concentrations divided by the predicted value, and the absolute median prediction error percent [12]. The former describes the bias and the latter the precision (variability) of the predictions.

\section{Results}

The clinical and demographic characteristics of the study group are summarized in Table 1. A total of 366 plasma vancomycin concentrations from 45 patients were included in the analysis. Of these, 26 patients were investigated prospectively, providing 227 (62\%) plasma concentrations. In nine of these patients plasma was sampled after a single dose administered following cannulation and once extracorporeal blood flow had been established, whereas in 19 patients sampling followed the first and fourth doses during the treatment of suspected or confirmed infections. Two patients were

Table 1

Clinical characteristics of study group

\begin{tabular}{|c|c|c|c|c|}
\hline & Overall & $\begin{array}{l}\text { Neonates } \\
\text { (0-1 month) }\end{array}$ & $\begin{array}{l}\text { Children } \\
\text { (1 month-18 years) }\end{array}$ & Adults (>18 years) \\
\hline Male & 29 & 8 & 9 & 12 \\
\hline Females & 16 & 7 & 3 & 6 \\
\hline Weight (kg) & $36.0(2.5-116)$ & $3.5(2.5-4.5)$ & $14.3(2.4-57)$ & $78.8(49.5-116)$ \\
\hline Sparse data group & 19 & 2 & 5 & 12 \\
\hline Rich data group & 26 & 13 & 7 & 6 \\
\hline \multicolumn{5}{|l|}{ Number of samples per patient } \\
\hline Sparse & $8.2(2-20)$ & $8(4-12)$ & $8(3-18)$ & $6.9(2-20)$ \\
\hline Rich & $7.3(4-12)$ & $5.8(4-12)$ & $8.1(4-12)$ & $9.5(5-12)$ \\
\hline$V A^{d}$ & 6 & 2 & 3 & 1 \\
\hline Undetermined & 6 & 1 & 5 & 0 \\
\hline Duration of ECMO (h) & $264.4(50-907)$ & $220.2(50-494)$ & $257.9(73-550)$ & 308.5 (86-907) \\
\hline \multicolumn{5}{|l|}{ Outcome } \\
\hline Survived & 27 & 11 & 5 & 11 \\
\hline Deceased & 18 & 4 & 7 & 7 \\
\hline
\end{tabular}

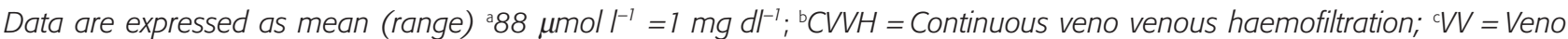
venous; ${ }^{\mathrm{V}} \mathrm{VA}=$ Veno arterial. 
excluded from the original data set because of untraceable medical records.

The prophylactic doses of vancomycin used were $15 \mathrm{mg} \mathrm{kg}^{-1}$ in neonates and children, and $1000 \mathrm{mg}$ in adults. For treatment of infections, initial doses ranged between 10 and $15 \mathrm{mg} \mathrm{kg}^{-1}$ every $6-24 \mathrm{~h}$ in children, and $750-1000 \mathrm{mg}$ every $12-24 \mathrm{~h}$ in adults. The overall mean (SD) peak and trough concentrations at steady state were 30.0 (10.1) and $11.9(6.8) \mathrm{mg} \mathrm{l}^{-1}$, respectively. (The reference range for serum vancomycin concentrations in this institution is trough $5-15 \mathrm{mg} \mathrm{l}^{-1}$, peak $20-30 \mathrm{mg}$ $\left.1^{-1}\right)$. All neonates in this study were term or near term with median (range) gestational age 40.4 (34.3-42) weeks. In those patients studied $(n=38)$, the majority (84\%) underwent veno-venous ECMO. Median (range) serum creatinine concentrations were significantly higher amongst adults (142.8 (48.3-224.5)), compared with neonates and older children (64.3 (39-180), and 68.8 (26.5-158.9), $\mu \mathrm{mol} \mathrm{l}^{-1}$, respectively). However, a greater number of children (42\%) were supported with CVVH than neonates or adults (13\% and 33\%, respectively).

The two compartment model was chosen in preference to that with one compartment, since it demonstrated a more appropriate structural model on examination of the graphical residual plots and a lower OFV. Although a three compartmental model resulted in a further lowering of the OFV, it was not explored further because the parameter estimates were imprecise, probably reflecting insufficient sampling to allow three compartments to be discerned. Residual error was best described by a combined additive and proportional structure. Significant improvement in model fit was achieved when weight was associated linearly (without an intercept) with each parameter, before investigation of other covariates. Allometric transformation of weight (by setting weight to a power of 0.75) was also tested, but did not improve the fit further. Plots of Bayesian posterior estimates of parameters from the basic model $v s$. the covariates revealed significant correlations (Figure 1), namely those between serum creatinine and clearance (CL), age and CL, central volume (V1) and intercompartmental clearance $(\mathrm{Q})$ and ECMO pump flow rates and $Q$. The significance of these relationships was investigated by hypothesis testing of full and reduced model during the covariate screening stage (Table 2). The largest decrease in OFV (97 U) was observed when serum creatinine was incorporated into a nonlinear model of $\mathrm{CL}\left(\mathrm{CL}=\theta_{1} \times \mathrm{Cr}^{\theta 2}\right.$, where $\theta$ is to be estimated). Since the estimate of $\theta_{2}$ was -0.9 , the model was simplified to $\theta_{1} / \mathrm{Cr}$, without a significant change in the OFV. Clearance was linearly related to age
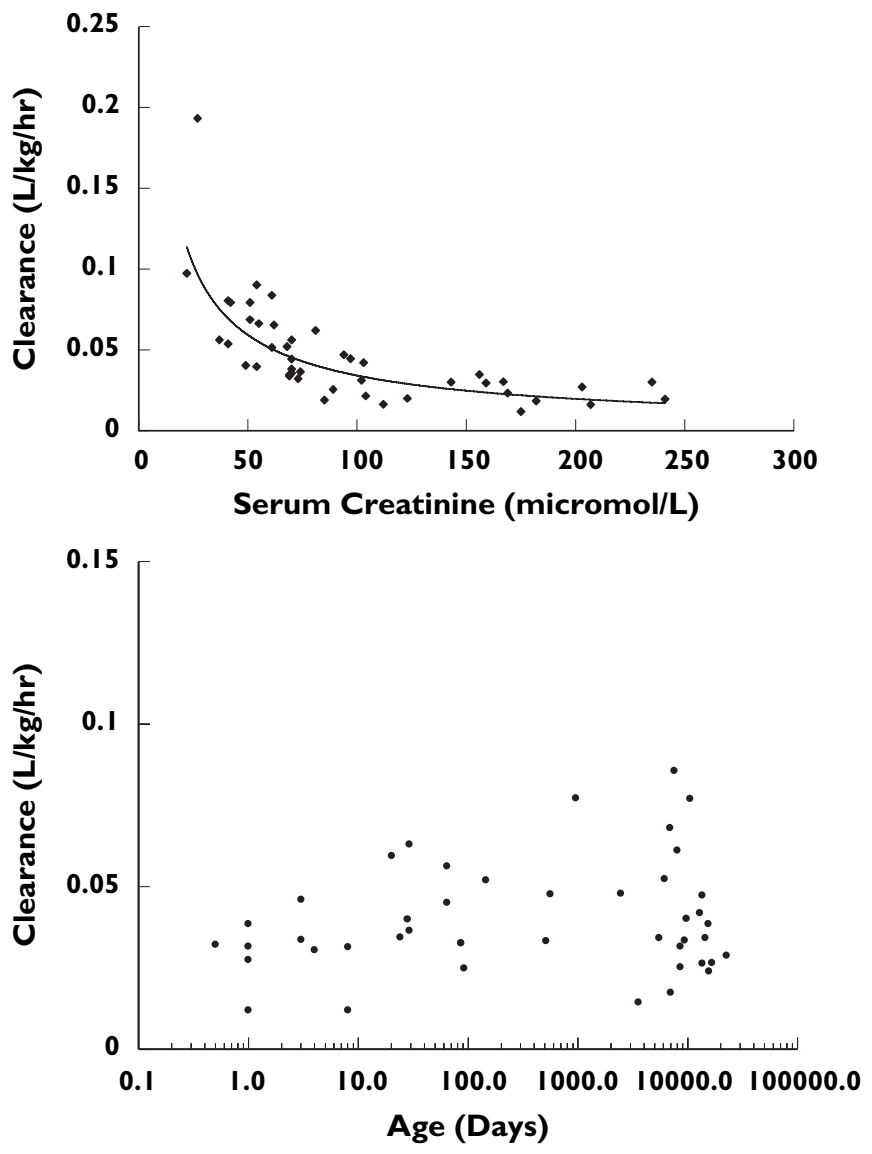

Figure 1

Plots of Bayesian posterior estimates of $\mathrm{CL}$ with covariates

in neonates and children up to 1000 days, but beyond this, was independent of age. Surprisingly, there was no significant difference in CL between those patients on and off CVVH $(P=0.183)$. Although $V 1$ decreased with age, there appeared to be a bimodal distribution with a break point at 4000 days. Thus the influence of age on $V 1$ when included into the model as a dichotomous variable resulted in a significant drop in OFV. Although the incorporation of an inverse relationship between age and Q in the model decreased OFV significantly, it was not included in the final model, since it had little influence on the scatterplots or interpatient variability. By the same rationale, the positive influence of ECMO pump flow rates on $\mathrm{Q}$ was also omitted from the final model.

The final structural model and parameter estimates are displayed in Table 3 . The relationship between observations and predictions from the basic \& final models are shown in Figure 2. Bias and precision of the respective models were $-0.8,-1.9 \%$ and $29.5,20.3 \%$. The mean Bayesian posterior estimates of CL, V1, volume of distribution at steady state $\left(V_{\mathrm{ss}}\right)$ and half-life 

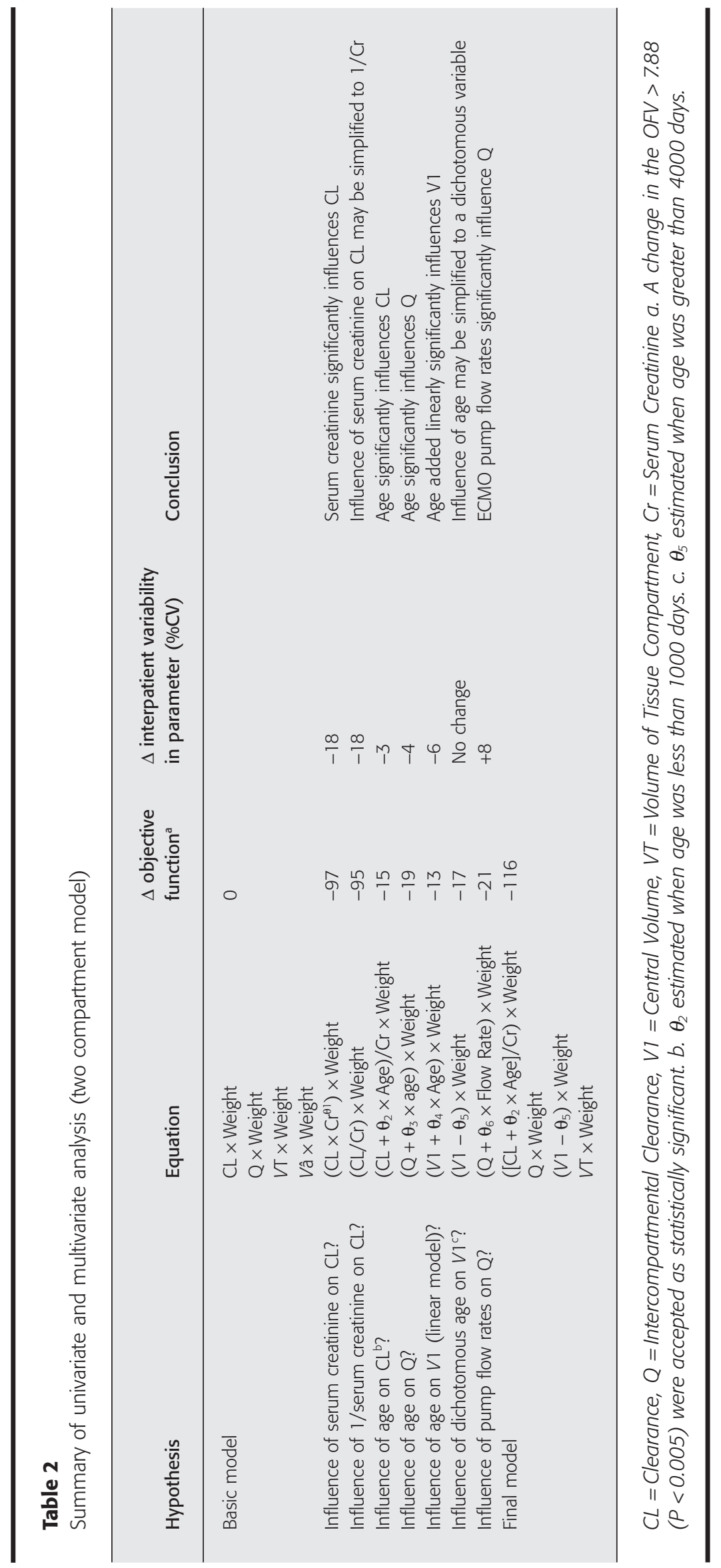


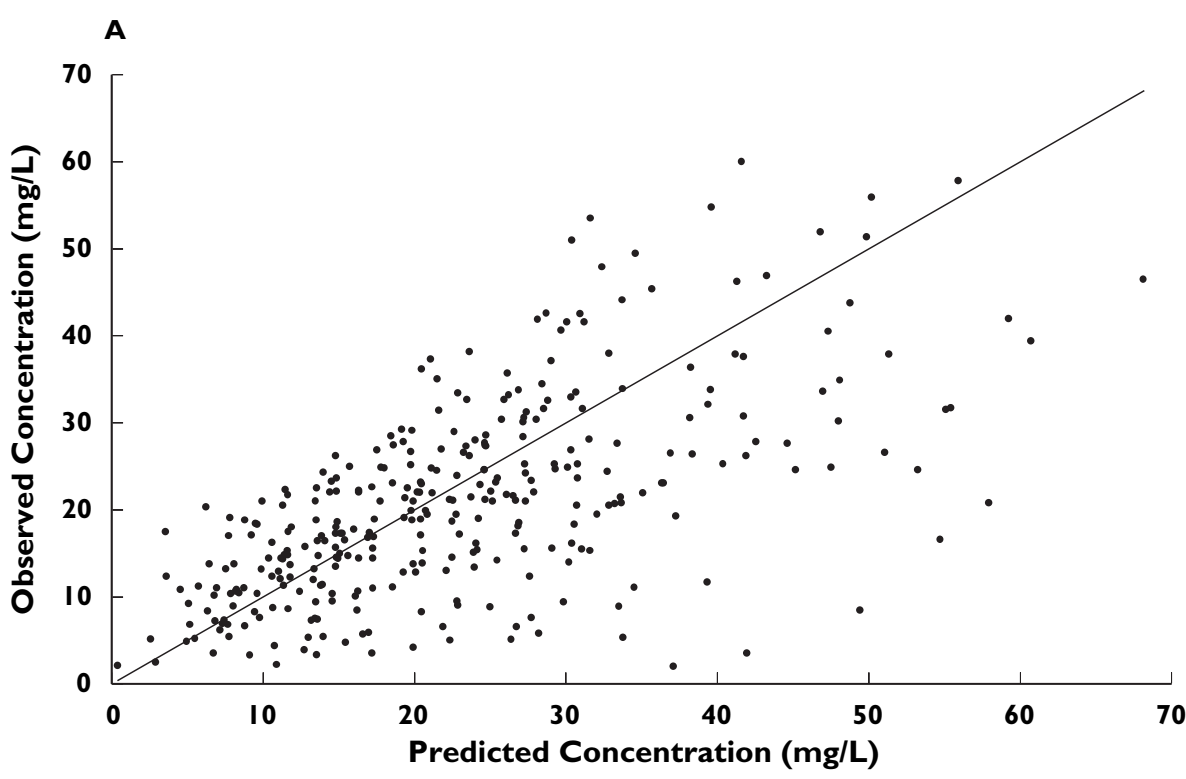

Figure 2

Scatterplot of observed versus predicted plasma vancomycin concentrations $(\mathrm{mg} / \mathrm{L})$ indicated a closer distribution around the line of unity for the final model (B) versus the basic model (A)

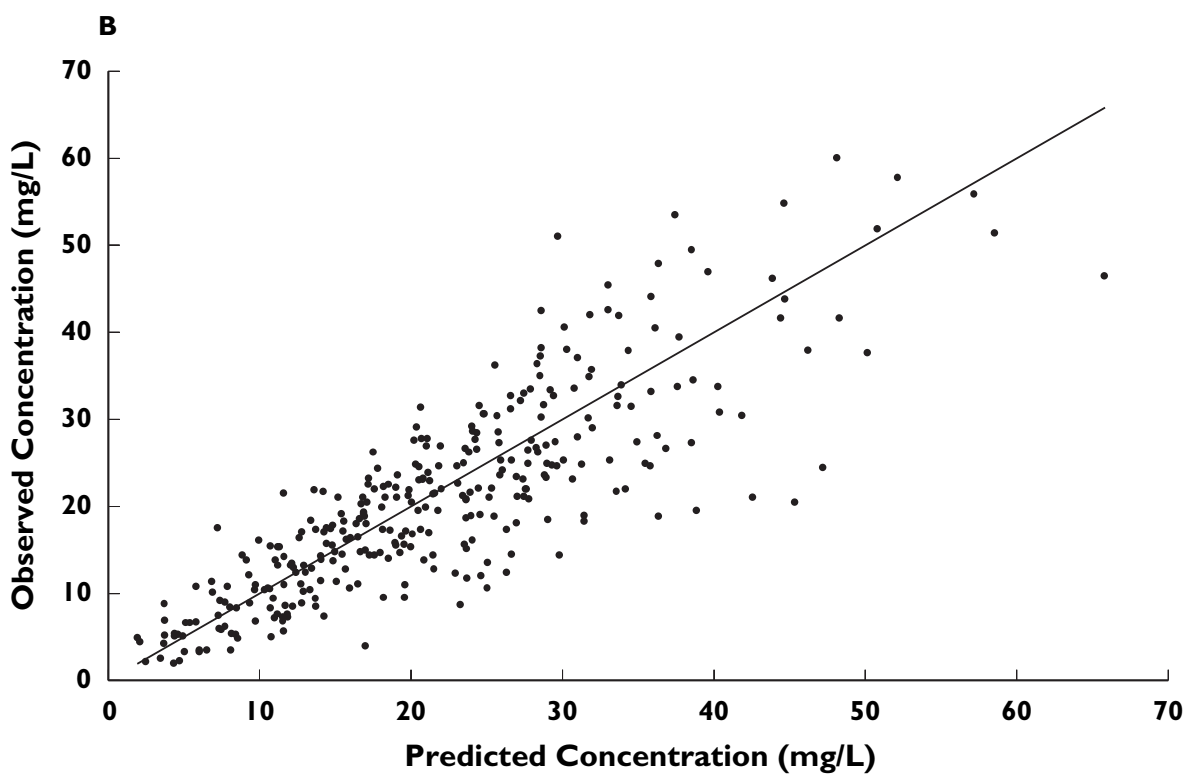

determined by the final model for each age category are displayed in Table 4. There were no significant differences between the age groups except for $V 1(P=0.006)$.

The 20 patients in the validation group (eight neonates, four children, eight adults) provided a total of 110 plasma concentrations for evaluation (Table 6). A scatter plot of residuals (observed minus predicted concentrations) against the predicted concentration revealed homogeneous scatter around the zero ordinate (Figure 3 ). The bias for the validation set was $-7.7 \%$, indicating that the model has a slight tendency to over predict whereas its precision was found to be $26.7 \%$.

The population parameter estimates from the final model were used to determine dosing regimens required to achieve approximate peak and trough concentrations of 30 and $10 \mathrm{mg} \mathrm{l}^{-1}$, respectively, for a range of age and serum creatinine values (Table 5). These and the final model parameters were used to simulate plasma drug concentration-time profiles which are displayed in Figure 4.

\section{Discussion}

Recent years have seen an increased usage of intravenous vancomycin in intensive care patients. Nephrotoxicity and ototoxicity associated with vancomycin was originally related to impurities in the preparation as well as high serum concentrations, but with better purification and improvements in formulation, the incidence of 
Table 3

Population pharmacokinetic parameter details for the final model of vancomcyin in ECMO patients

\begin{tabular}{|c|c|c|}
\hline & Estimate & Standard error \\
\hline \multirow[t]{2}{*}{$\mathrm{CL}\left(\mid \mathrm{kg}^{-1} \mathrm{~h}^{-1}\right)$} & $\begin{array}{l}{[2.4+0.0018 \times \text { Age }(\text { days })] / C r\left(\mu \mathrm{mol} \mathrm{I}^{-1}\right),} \\
\text { if Age }<1000 \text { days }\end{array}$ & 0.18 \\
\hline & $4.3 / \mathrm{Cr}\left(\mu \mathrm{mol} \mathrm{I}^{-1}\right)$, if Age $>1000$ days & 0.24 \\
\hline $\mathrm{Q}\left(\mid \mathrm{kg}^{-1} \mathrm{~h}^{-1}\right)$ & 0.09 & 0.023 \\
\hline \multirow[t]{2}{*}{$V 1\left(1 \mathrm{~kg}^{-1}\right)$} & 0.45 if Age $<4000$ days, & 0.038 \\
\hline & 0.37 if Age $>4000$ days & 0.042 \\
\hline$V T\left(\mid \mathrm{kg}^{-1}\right)$ & 0.25 & 0.037 \\
\hline \multicolumn{3}{|c|}{ Population variability ${ }^{\mathrm{a}}$} \\
\hline $\mathrm{CL}$ & $25 \%$ & \\
\hline$V_{1}$ & $25 \%$ & \\
\hline Q & $91 \%$ & \\
\hline$V T$ & $48 \%$ & \\
\hline Residual error & $12.1 \% \pm 2.1 \mathrm{mgl}^{-1}$ & \\
\hline
\end{tabular}

aExpressed as coefficient of variation.

Table 4

Summary of Bayesian posterior parameter estimates in the age related categories

\begin{tabular}{|c|c|c|c|c|c|}
\hline V1 $\left(1 \mathrm{~kg}^{-1}\right)$ & $0.42(0.1)$ & $0.45(0.1)$ & $0.46(0.08)$ & $0.37(0.08)$ & $P=0.006$ \\
\hline $\mathrm{CL}\left(\mid \mathrm{kg}^{-1} \mathrm{~h}^{-1}\right)$ & $0.05(0.03)$ & $0.04(0.02)$ & $0.06(0.04)$ & $0.04(0.02)$ & $P=0.37$ \\
\hline Half-life (h) & $8.44(5.17)$ & $10.40(6.67)$ & $6.18(3.07)$ & $8.55(4.64)$ & $P=0.1$ \\
\hline
\end{tabular}

Data are expressed as mean (SD).

Table 5

Dosing guidelines

Dose $\left(\mathrm{mg} \mathrm{kg}^{-1}\right) /$ Interval (h)

\begin{tabular}{|c|c|c|c|c|c|}
\hline \multirow{2}{*}{$\begin{array}{l}\text { Serum creatinine } \\
\left(\mu \mathrm{mol} \mathrm{I}{ }^{-1}\right)\end{array}$} & \multirow[b]{2}{*}{1} & & \multicolumn{3}{|c|}{ Age (days) } \\
\hline & & 365 & 1000 & $1000-4000$ & $>4000$ \\
\hline 25 & $15 / 8$ & $20 / 8$ & $20 / 6$ & $20 / 6$ & $20 / 6$ \\
\hline 50 & $10 / 12$ & $10 / 8$ & $10 / 8$ & $10 / 6$ & $10 / 6$ \\
\hline 100 & $10 / 24$ & $15 / 24$ & $10 / 12$ & $15 / 18$ & $10 / 12$ \\
\hline 150 & $15 / 48$ & $15 / 36$ & $12.5 / 24$ & $15 / 24$ & $12.5 / 24$ \\
\hline 200 & Re dose after concentrations, approx. every $72 \mathrm{~h}$ & $15 / 48$ & $15 / 36$ & $15 / 36$ & $15 / 36$ \\
\hline
\end{tabular}


Table 6

Clinical and demographic characteristics of validation group

\begin{tabular}{|c|c|c|c|c|}
\hline & Overall & Neonates & Paediatric & Adult \\
\hline Number of patients & 20 & 8 & 4 & 8 \\
\hline Weight (kg) & $42.4(44.5)$ & $3.6(0.6)$ & $21.9(23.0)$ & $91.4(20.7)$ \\
\hline Age at cannulation (years) & $16.9(20.4)$ & 5.5 (8.8) days & $6.0(7.1)$ & $39.2(12.1)$ \\
\hline Cannulation (VV/VA) & $18 / 2$ & $7 / 1$ & $3 / 1$ & $8 / 0$ \\
\hline Serum creatinine $\left(\mu \mathrm{mol} \mathrm{I}^{-1}\right)$ & $111.5(74.1)$ & $65.6(34.4)$ & $56.9(17.9)$ & $157.3(79.6)$ \\
\hline Number of samples per patient & $5.4(3.1)$ & $4.9(4.2)$ & $4.3(1.5)$ & $6.5(2.3)$ \\
\hline
\end{tabular}

Data are expressed as mean (SD).

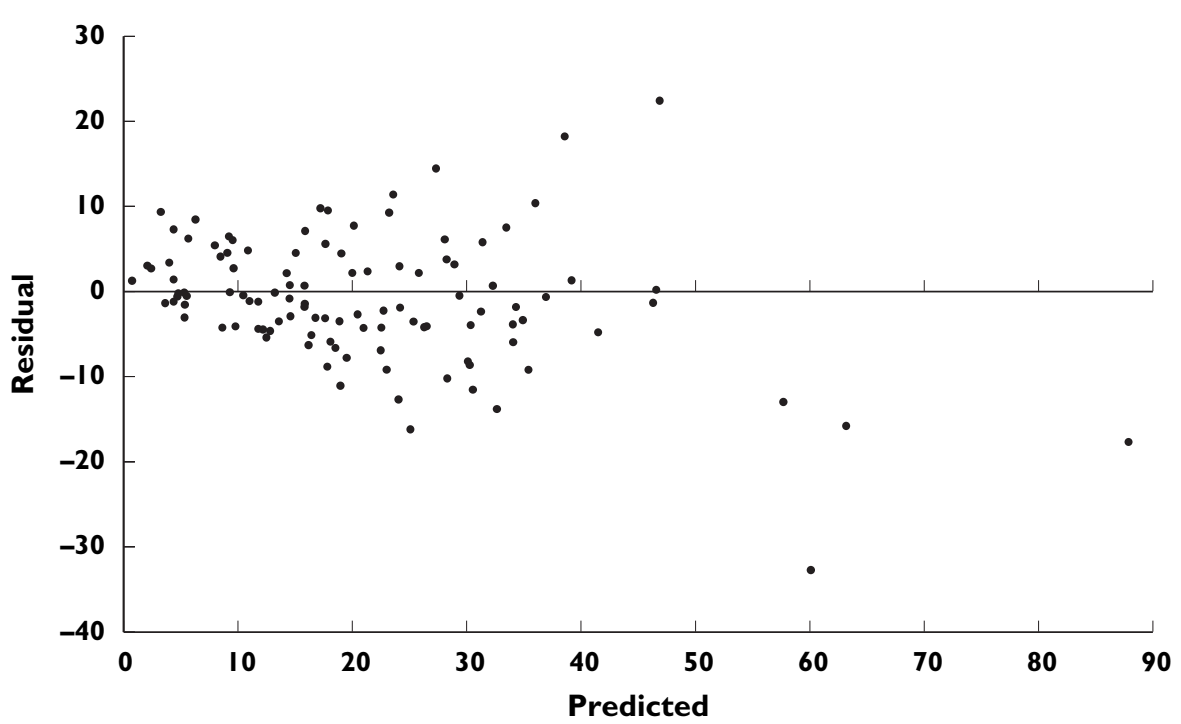

Figure 3

Assessment of predictive performance of final population model in a separate set of 20 patients (110 observations): Plot of residual (observed minus predicted) versus predicted plasma vancomycin concentration toxicity is not thought to be as prevalent [13]. There are no definitive studies relating toxicity to serum vancomycin concentrations, raising questions as to whether or not there is a need to monitor serum values [14]. However, since vancomycin efficacy is related to the maintenance of plasma concentrations above a minimum threshold value for the duration of therapy, monitoring of trough concentrations is still considered necessary, particularly in critically ill patients exhibiting wide interpatient variability [15-17]. Although pharmacokinetic data for vancomycin have become more readily available in this population, there have been limited and contradictory reports in patients undergoing ECMO.

Hoie et al. investigated vancomycin pharmacokinetics in six term neonates and concluded that their findings were similar to previous reports from nonECMO infants [6]. However, the dosage regimen of $20 \mathrm{mg} \mathrm{kg}^{-1} 18$ hourly that they propose in patients with normal renal function suggests a decreased clearance and an enlarged volume of distribution. In contrast, Amaker et al. investigated 12 infants undergoing ECMO (mean gestational age 39 weeks, mean serum creatinine $\left.99 \mu \mathrm{mol} \mathrm{l}^{-1}\right)$, revealing a larger $V_{\mathrm{ss}}($ mean $\pm \mathrm{SD} ; 1.06 \pm$ $\left.0.451 \mathrm{~kg}^{-1}\right)$, a lower clearance $\left(0.047 \pm 0.0111 \mathrm{~h}^{-1} \mathrm{~kg}^{-1}\right)$ and a prolonged $t_{1 / 2, \mathrm{z}}(16.9 \pm 9.5 \mathrm{~h})$ [7]. Buck compared ECMO patients $(n=15)$ with a control group $(n=15)$ utilizing steady state peak and trough measurements, and found no significant differences in volume and clearance but a longer half-life in ECMO patients $(8.29 \pm 2.23$ vs. $6.53 \pm 2.05 \mathrm{~h})$ [8]. A recent population 

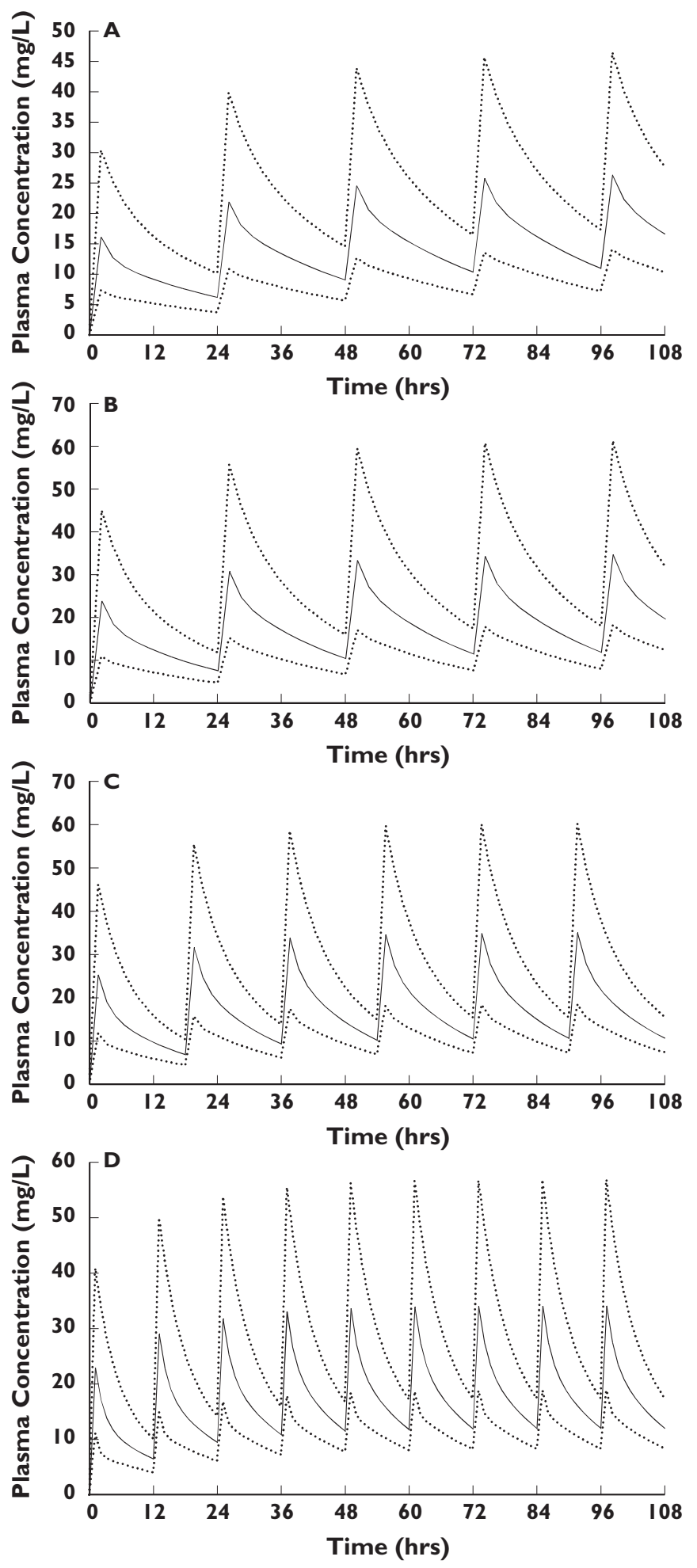

Figure 4

Simulated mean plasma profiles and 95\% Confidence Intervals of Interindividual Variability in an age range ECMO population with serum creatinine of 100 micromol/L. (a) Neonate ( 1 day), $10 \mathrm{mg} / \mathrm{kg}$ every 24 hours (b) Child (1 year), 15 mg/kg every 24 hours (c) Child (10 years), $15 \mathrm{mg} / \mathrm{kg}$ every 18 hours (d) Adult, $10 \mathrm{mg} / \mathrm{kg}$ every 12 hours. study of 374 infants identified the small number $(n=15)$ of ECMO patients as displaying an enlarged volume and decreased clearance, although not significantly so, once serum creatinine and gestational age had been included in the final model [18].

Though the present study was not controlled, comparison of the data with those previously reported in similar non-ECMO patients suggests that the pharmacokinetics of vancomycin are altered. The current study population had wide, age range related, serum creatinine values. In total, 10 neonates $(66 \%)$ and five children $(42 \%)$ had concentrations above $60 \mu \mathrm{mol} \mathrm{l}^{-1}$, whereas 11 adults (61\%) exhibited values greater than $120 \mu \mathrm{mol} \mathrm{l}^{-1}$. These serum creatinine concentrations reflect the critically ill nature of these patients, who are likely to have suffered hypoxic ischaemic renal injury prior to the initiation of ECMO. A high proportion of patients (29\%) also required CVVH support. Similar to previous population studies in non-ECMO patients, vancomycin clearance was found to be strongly associated with renal function [18-20].

Caparelli et al. determined the clearance of vancomycin in a typical study infant (serum creatinine = $53 \mu \mathrm{mol}{ }^{-1}$, weight $=1.8 \mathrm{~kg}, \mathrm{GA}=33.5$ weeks, age $=$ 27 days) to be $0.0661 \mathrm{~kg}^{-1} \mathrm{~h}^{-1}$ [18]. The mean Bayesian posterior estimate of clearance for term neonates in the present study (serum creatinine $=64.3 \mu \mathrm{mol} \mathrm{l}{ }^{-1}$, weight $=3.4 \mathrm{~kg}, \mathrm{GA}=39.8$ weeks, age $=1$ day) was $0.04 \mathrm{l} \mathrm{kg}^{-1} \mathrm{~h}^{-1}$, a value somewhat lower than previously reported. Two previous studies of infants and children reported clearances of $0.071 \mathrm{~kg}^{-1} \mathrm{~h}^{-1}$ and $0.08 \mathrm{l} \mathrm{kg}^{-1} \mathrm{~h}^{-1}$ in term neonates $[21,22]$. An increase in clearance with age (up to 1000 days) was observed and this is in line with the maturation of kidneys. Although renal clearance is low in the neonate, it increases rapidly with age such that weight adjusted total body clearance in children is considerably higher [23]. Previous studies have reported weight adjusted total body clearance of vancomycin to be $2-3$ times higher in the paediatric population $\left(0.281 \mathrm{~kg}^{-1} \mathrm{~h}^{-1}\right)$ compared with adults $\left(0.081 \mathrm{~kg}^{-1}\right.$ $\left.\mathrm{h}^{-1}\right)[22,24]$. The mean Bayesian posterior estimate of clearance for children and adults on ECMO in the present study were substantially lower at 0.06 and $0.04 \mathrm{l} \mathrm{kg}^{-1} \mathrm{~h}^{-1}$, respectively. Allometric transformations of weight associated with clearance did not improve the model, possibly due to neonates confounding the simple relationship since kidneys do not fully mature until approximately 6 months of age [23]. In the present study, CVVH did not appear to influence clearance or other parameters, suggesting that serum creatinine is a more important indicator of vancomycin clearance even during CVVH. One reason for this may be that $\mathrm{CVVH}$ 
is often implemented for managing fluid balance (particularly at the onset of ECMO) rather than in response to acute renal failure.

The expanded circulating blood volume during ECMO is expected to increase the volume of distribution for a water soluble molecule like vancomycin and thus decrease its elimination. Increases in total blood volume are related to ECMO circuit size and range between 25\% (adults) and 125\% (neonates) [4]. This was reflected in the population estimates of $V 1$, which were found to be higher in neonates and younger children (age $<4000$ days) compared with older children and adults $\left(0.45 v s .0 .361 \mathrm{~kg}^{-1}\right.$, respectively). The estimate of $V_{\mathrm{ss}}\left(0.71 \mathrm{l} \mathrm{kg}^{-1}\right)$ in the present study is less than previously reported in ECMO infants by Amaker et al. $\left(1.06 \mathrm{l} \mathrm{kg}^{-1}\right)$, but higher than that in non-ECMO studies $\left(0.38-0.641 \mathrm{~kg}^{-1}\right)[21,22,24]$. Changes in volume of distribution may not be due simply to the extra circulating volume, but also to increased extracellular fluid and increased renin and atrial natriuretic peptide release secondary to nonpulsatile renal blood flow during venoarterial ECMO [25].

The validation exercise demonstrated that the model had good precision and was reasonably unbiased. Dosing guidelines developed using parameter estimates from the final model indicate the influence of two significant covariates, age and serum creatinine. Using these dosing regimens, plasma concentration profiles and associated $95 \%$ confidence intervals of the population variability were simulated. Since trough plasma vancomycin concentrations are important for antimicrobial efficacy, the aim was to maintain trough concentrations over the range 10 to $15 \mathrm{mg} \mathrm{l}^{-1}$, and to avoid the lower and upper confidence intervals falling outside 5 and $20 \mathrm{mg} \mathrm{l}^{-1}$, respectively [26]. Although mean plasma concentration profiles obtained in all simulations were acceptable, the confidence intervals indicate the difficulty in predicting vancomycin pharmacokinetics in an individual, a finding that reinforces the importance of therapeutic drug monitoring. Finally, for neonates in the first few days of life, serum creatinine is reflective of maternal derived creatinine and thus renal function may be underestimated. Thus, the starting doses suggested may result in sub therapeutic plasma concentrations and particular attention should be paid to individualization of doses in these circumstances.

In a study group consisting of the full spectrum of term neonates, older children and adults with a wide range of primary diagnoses, a population pharmacokinetic model was developed for vancomycin during ECMO, based on serum creatinine and age. Parameter estimates reveal significantly decreased vancomycin clearance and increased volume of distribution when compared with critically ill non-ECMO patients of similar age and suggest altered disposition during ECMO. The pharmacokinetic parameter estimates were used to develop a guide to vancomycin dosing for those involved in the care of this specialist group of critically ill patients.

This study was funded by the Department of ECMO, Glenfield Hospital, Leicester.

\section{References}

1 Bartlett R, Roloff D, Custer J, Younger J, Hirschl R. Extracorporeal life support. The University of Michigan Experience. J Am Med Assoc 2000; 283: 904-8.

2 Peek G, Killer H, Sosnowski A, Firmin R. Extracorporeal membrane oxygenation: potential for adults and children? (Review). Hospital Med (London) 1998; 59: 304-8.

3 Mulla H, Lawson G, Firmin R, Upton D. Drug disposition during extracorporeal membrane oxygenation (ECMO). Paediatric Perinatal Drug Ther 2001; 4: 109-20.

4 Mulla H, Nabi F, Nichani S, Lawson G, Firmin R, Upton D. Population pharmacokinetics of theophylline during paediatric extracorporeal membrane oxygenation. Br J Clin Pharmacol 2003; 55: $23-31$.

5 Mulla $\mathrm{H}$, McCormack $\mathrm{P}$, Lawson G, Firmin R, Upton D. Pharmacokinetics of midazolam in neonates undergoing extracorporeal membrane oxygenation. Anesthesiology; (in press).

6 Hoie E, Swigart S, Leuschen M. Vancomycin pharmacokinetics in infants undergoing extracorporeal membrane oxygenation. Clin Pharmacokinet 1990; 9: 711-5.

7 Amaker R, DiPiro J, Bhatia J. Pharmacokinetics of vancomycin in critically ill infants undergoing extracorporeal membrane oxygenation. Antimicrobial Agents Chemother 1996; 40: 113942

8 Buck M, Ksenich R, Wooldridge P. Vancomycin pharmacokinetics in neonates receiveing extracorporeal membrane oxygenation. Pharmacotherapy 1998; 18: 1082.

9 White LO, Holt HA, Reeves DS, MacGowan A. Evaluation of Innufluor fluorescence polarisation immunoassay kits for the determination of serum concetrations of gentamicin, tobramycin, amikacin and vancomycin. J Antimicrobial Chemother 1997; 39: 355-61.

10 Pharsight. Winnonmix Professional. In 2.0.1. eds. California 94040: Pharsight Corporation. 1993-2000.

11 Compaq. Compaq Visual Fortran Professional Edition. In 6.5., eds. Compaq Computer Corporation; September 2000.

12 Sheiner L, Beal S. Some suggestions for measuring predictive performance. J Pharmacokin Biopharm 1981; 9: 503-12.

13 Odio C, McCracken GJ, Nelson J. Nephrotoxicity associated with vancomycin-aminoglycoside therapy in four children. J Pediatrics 1984; 105: 491-3. 
14 Bhatt-Mehta V, Schumacher R, Faix R, Leady M, Brenner T. Lack of vancomycin-associated nephrotoxicity in newborn infants: a case controlled study. Pediatrics 1999; 103: e48.

15 Lowdin E, Odenholt I, Cars O. In vitro studies of pharmacodynamic properties of vancomycin against Staphylococcus aureus and Staphylococcus epidermidis. Antimicrobial Agents Chemother 1998; 42: 2739-44.

16 Duffull S, Begg E, Chambers S, Barclay M. Efficacies of different vancomycin dosing regimens against Staphylococcus aureus determined with a dynamic in vitro model. Antimicrobial Agents Chemother 1994; 38: 2480-2.

17 Zimmermann A, Katona B, Plaisance K. Association of vancomycin serum concentrations with outcomes in patients with grampositive bacteremia. Pharmacotherapy 1995; 15: 85-91.

18 Capparelli E, Lane J, Romanowski G, McFeely E, Murray W, Sousa $\mathrm{P}$, Kildoo $\mathrm{C}$. The influences of renal function and maturation on vancomycin elimination in newborns and infants. J Clin Pharmacol 2001; 41: 927-34.

19 Yasuhara M, Iga T, Zenda H, Okumura K, Oguma T, Yano Y, Hori R. Population pharmacokinetics of vancomcyin in Japanese adult patients. Ther Drug Monit 1998; 20: 139-48.
20 Grimsley C, Thomson A. Pharmacokinetics and dose requirements of vancomycin in neonates. Arch Dis Childhood Fetal Neonatal Edition 1999; 81: F221-F227.

21 Naqvi S, Keenan W, Reichley R, Fortune K. Vancomcyin pharmacokinetics in small, seriously ill infants. Am J Dis Childhood 1986; 140: 107-10.

22 Schaad U, McCracken G, Nelson J. Clinical pharmacology and efficacy of vancomcyin in pediatric patients. J Pediatrics 1980; 96 : 119-26.

23 Rowland M, Tozer T. Clinical pharmacokinetics. Concepts and applications, 3rd edn. Philadelphia: Lippincott. Williams \& Wilkins 1995.

24 Rodvold K, Blum R, Fischer J, Zokufa H, Rotschafer J, Crossley K, Riff $L$. Vancomcyin pharmacokinetics in patients with various degrees of renal function. Antimicrobial Agents Chemotherapy 1988; 32: 848-52.

25 Bartlett R. Extracorporeal life support for cardiopulmonary failure. Current Problems Surg 1990; 27: 621-705.

26 de Hoog M, Schoemaker R, Mouton J, van den Anker J. Vancomycin population pharmacokinetics in neonates. Clin Pharmacol Therapeutics 2000; 67: 360-7. 